\title{
The Exchange of Translation Tools
}

Gülfidan Aytaş *

* Sakarya Üniversitesi

E-mail: gayvaz@sakarya.edu.tr

Copyright (C) 2016 Gülfidan Aytaş. This is an open access article distributed under the Eurasian Academy of Sciences License, which permits unrestricted use, distribution, and reproduction in any medium, provided the original work is properly cited.

\begin{abstract}
Translator and interpreter have received today new tools with the evolution of electronical instruments and multi-directional communication technologies. The tools used especially as communication medias have been utilized today consummately on translation. Hence, in this study are linked up the tools for translation with mass communication medium. The aim of this study is to state the conventional translation tools and modern tools and besides to dispute the growing of these tools and their impact on translation. Eventually it is here mentioned that the role of translator has exchanged by means of these tools und his responsibilities go up not only as a text producer but also as a investigator.
\end{abstract}

Keywords: Translation, tools, technology, translation process

\section{Der Wandel der Übersetzungshilfsmittel (Çeviri Araçlarının Değişimi)}

\section{ÖZET}

Günümüzde, çevirmenler ve tercümanlar, elektronik araçların gelişmesi ve çok yönlü iletişim teknolojilerinde yaşanan gelişmeler ile birlikte yeni çeviri araçlarına kavuşmuşlardır. Özellikle iletişim alanında başvurulan yardımcı araçlar da bugün çeviri eylemi esnasında kullanılmaktadır. Bu sebeple, bu makalede kitle iletişim bağlamında yer verilen çeviriye yardımcı araçlar ele alınacaktır. Makalenin amacı, geleneksel çeviri araçlarını ve günümüzdeki çeviriye yardımcı araçları sıralayarak onların kullanımını ve çeviri süreci üzerindeki etkisini göstermektir. Son olarak, sadece metin üreticisi değil aynı zamanda bir aktarıcı göreviyle yeni çeviri araçlarını tanımak zorunda olan çevirmenin değişen rolünden bahsedilecektir.

Anahtar Kelimeler: Çeviri, yardımcı araçlar, teknoloji, çeviri süreci

\section{Einleitung}

Der folgende Aufsatz behandelt einige Veränderungen von der Enstehung der Übersetzung bis zum heutigen Übersetzungsfaktum. Die Informatik und technologischen Kenntnisse, die ständig aktualisiert werden, ermöglichen zugleich die Verkörperung der Übersetzungshandlung. In diesem Zusammenhang ist das Subjekt, welches zunächst in dieser Arbeit vermittelt werden soll, die Übersetzungsanschauung in der Anwendung, die sogenannte 
traditionelle Übersetzung. Dabei ist es wichtig $\mathrm{zu}$ untersuchen, wie sich der Übersetzungsprozess erfüllt und welche Nachschlagewerke vom Übersetzer nachgesucht werden. Hinsichtlich der vergangenen Übersetzungsanschauung werden die Hilfsmittel, die der Übersetzer während des Übersetzungsprozess benutzt, untersucht. Es wird hier angenommen, dass diese Hilfsmittel im Zusammenhang mit technologischen Anwendungen eine neue Bedeutung gewinnen und sich positiv auf den Übersetzungsprozess auswirken.

Es wird durch die Entfernung der traditionellen Übersetzungsauffassung damit begonnen, in einen neuen Übersetzungsprozess hineinzugehen. Indem die Faktoren, die es diesem Prozess ermöglichen $\mathrm{zu}$ entstehen, analysiert werden, werden die den Wandel der Übersetzungshilfsmittel beeinflussenden Parameter behandelt. Die Veränderungen, die die herkömmliche Übersetzungsauffassung erfährt, geben hiermit die Möglichkeit, dass die Übersetzungshilfsmittel sich von den traditionellen Eigenschaften befreien. Gleichzeitig mit der Entwicklung der Kommunikationmittel und neuen Aspekten entstehen für den Übersetzer nützliche Veränderungen, die eine Verbesserung und Erweiterung seiner Arbeit ermöglichen. Diese Kontinuität bewirkt, dass sich die Ausstattung und Vielseitigkeit der Übersetzungswerkzeuge in umfangreichem Maße verbessert.

Es wird die heutige Stellung solcher Übersetzungswerkzeug und die Art der Hilfsmittel, die man beim Übersetzen benutzt, sowie der Einfluss, den sie auf den Übersetzungsprozess haben, besprochen. Es ist das Ziel, mit der Veränderung der Übersetzungsmittel die nachher bestehende Form der Übersetzungshandlung zu beleuchten, die unmöglich vorauszusehen ist. Wenn analysiert werden kann, worin die Kommunikation zwischen den Menschen durch technologischen und wissenschaftlichen Wandel verändert wird, kann man hier auch sagen, wie der Übersetzungsprozess schneller und die Übersetzungsarbeit effektiver wird.

Die traditionelle Übersetzungsauffassung und der traditionelle Übersetzungsprozess hatten in der Phase, in der es die heutigen Möglichkeiten nicht gab, ihre Berechtigung. Um den Einfluss der sich verändernden Übersetzungswerkzeuge auf den Übersetzungsprozess zeigen zu können, ist es eine mögliche Methode, die in dieser Phase genutzten und die heutigen Übersetzungswerkzeuge gegenüber zu stellen sowie den traditionellen und den heutigen Übersetzungsprozess zu untersuchen.

\section{Die herkömmliche Übersetzung und Hilfsmittel in diesem Zeitraum}

Die Übersetzungshandlung besteht aus zwei Phasen, die der mündlichen und schriftlichen Überlieferung. Die mündliche Überlieferung gehört vor allem dem Zeitraum an, in dem es keine schriftlichen Werke gab. Die zu diesem Zeitraum gehörenden Übersetzungen wurden meistens von Fachleuten, Dichtern oder Philosophen verwirklicht. Die Bevölkerung erreichte ihr Wissen durch die Leute, die auf einem Platz einer grossen Menschenmenge gegenüber ihre Ideen von der Welt vorlegten. Darum waren die Übersetzungen eng auf ihre Aussagen und Ansätze beschränkt.

Die Übersetzungshandlung fand hier in einem Prozess statt, der schon seit der Antike gemäß den Möglichkeiten jener Zeit zustande gebracht wurde. Die Übermittler der Nachrichten wurden als Bote bezeichnet. Während sich die Übersetzung zunächst dadurch auszeichnete, dass sie die Kommunikation zwischen den Menschen verschiedener Sprachräume ermöglichte, hat sie sich mit der Zeit von einem Mittler zu einer zielorientierten Handlung gewandelt. Jetzt muss der diese Handlung erfüllende Übersetzer seine Recherchenachschlagewerke und Hilfsmittel aktualisieren. 
In den Jahren, in der die Übersetzung als eine Handlung gesehenen wurde, hat der Übersetzungsprozess damit angefangen, dass der Übersetzer den Ausgangstext durch einen Bekannten bekam. Dann benutzte der Übersetzer ein Wörterbuch oder die Enzyklopädien, um den Text zu bearbeiten. Wenn es jemanden gab, der den Übersetzungstextinhalt bereits bearbeitet hatte, wendete er sich an diesen.

Wenn der Übersetzer einen Text, der nicht mit seinem Wissensbereich übereinstimmt, übersetzt, dann muss er auch entweder umständliche Erklärungen mitliefern oder den Text mit semantischer Übersetzung in die Zielsprache übertragen. Der Übersetzer ist während dieses Prozesses nicht nur ein Sprachmittler, sondern auch eine Autorität, die den Ausgangstext und die Ausgangskultur analysieren kann, die Terminologie der Ausgangssprache beherrscht, das Terminologieäquivalent in der Zielsprache kennt, ein Kulturmittler, ein Fachmann oder eine Fachfrau bei der Terminologie, ein Dichter, Soziologe, Psychologe, sogar ein Textproduzent, der der Textfunktion gemäße Texte verfasst. Dass der traditionelle Übersetzungsprozess keine umfangreichen und vielfältigen Möglichkeiten enthalten hat, bedingte einen vielseitigen Übersetzer und gab dem Übersetzer eine große Verantwortung. Diese große Verantwortung, der er sich gegenüber sah, führte dazu, dass der Übersetzungsprozess länger dauerte sowie die Qualität litt und die Übersetzungshandlung stagnierte. Die Stagnation beim Übersetzen versucht man mit den traditionellen Hilfsmittel zu vermindern, aber weil dies unter den früheren Bedingungen nicht möglich war, kam der Prozess zum Stillstand.

Nach Şahin ist ein Übersetzer, der an dem Arbeitstisch mit vorhandenen Wörterbüchern und Enzyklopädien arbeitet, wenn nötig, seine Umgebung über bestimmte Themen zu Rate zieht und mit Papier und Stift seine Übersetzung vollendet, dem Begriff der herkömmlichen Übersetzung zuzuordnen(Şahin, 2013: 3).

$\mathrm{Zu}$ dieser Definition kann man noch hinzufügen, dass die Möglichkeiten einer bestimmten Zeitspanne auf den Übersetzungsprozess Einfluss nehmen, sich die Übersetzungshilfsmittel somit durch die Gelegenheit gestalten.

Um die Entwicklungen beim herkömmlichen Übersetzen $\mathrm{zu}$ untersuchen, ist es zuerst erforderlich, die Gelegenheiten und Möglichkeiten in schriftlicher und mündlicher Übersetzung und dann die Übersetzungsbildung zu analysieren.

\subsection{Die schriftliche Übersetzung}

Nach der Erfindung des Schreibens erlebte man erheblichen Entwicklungen in Wissenschaft und Technik. Diese Entwicklungen zeigen uns die Veränderung der früher bestehenden Übersetzungshilfsmittel.

Während der Übersetzer seine Kompetenzen und Fertigkeiten beim Übersetzen benutzt, entwickelt er auch seine Übersetzungspraxis mithilfe äußerer Einflüsse.

In der Zeit vor dem mit den technologischen und wissenschaftlichen Entwicklungen aufgetauchten Begriff "Internet" bestanden die Übersetzungshilfmittel meistens aus schriftlichen Nachschlagewerken und Fachleuten. Folglich kann man die folgende Abbildung für die Übersetzungskommunikation und -hilfsmittel als ein Beispiel ansehen. 
Die Hilfsmitteltypologie von B. Nord

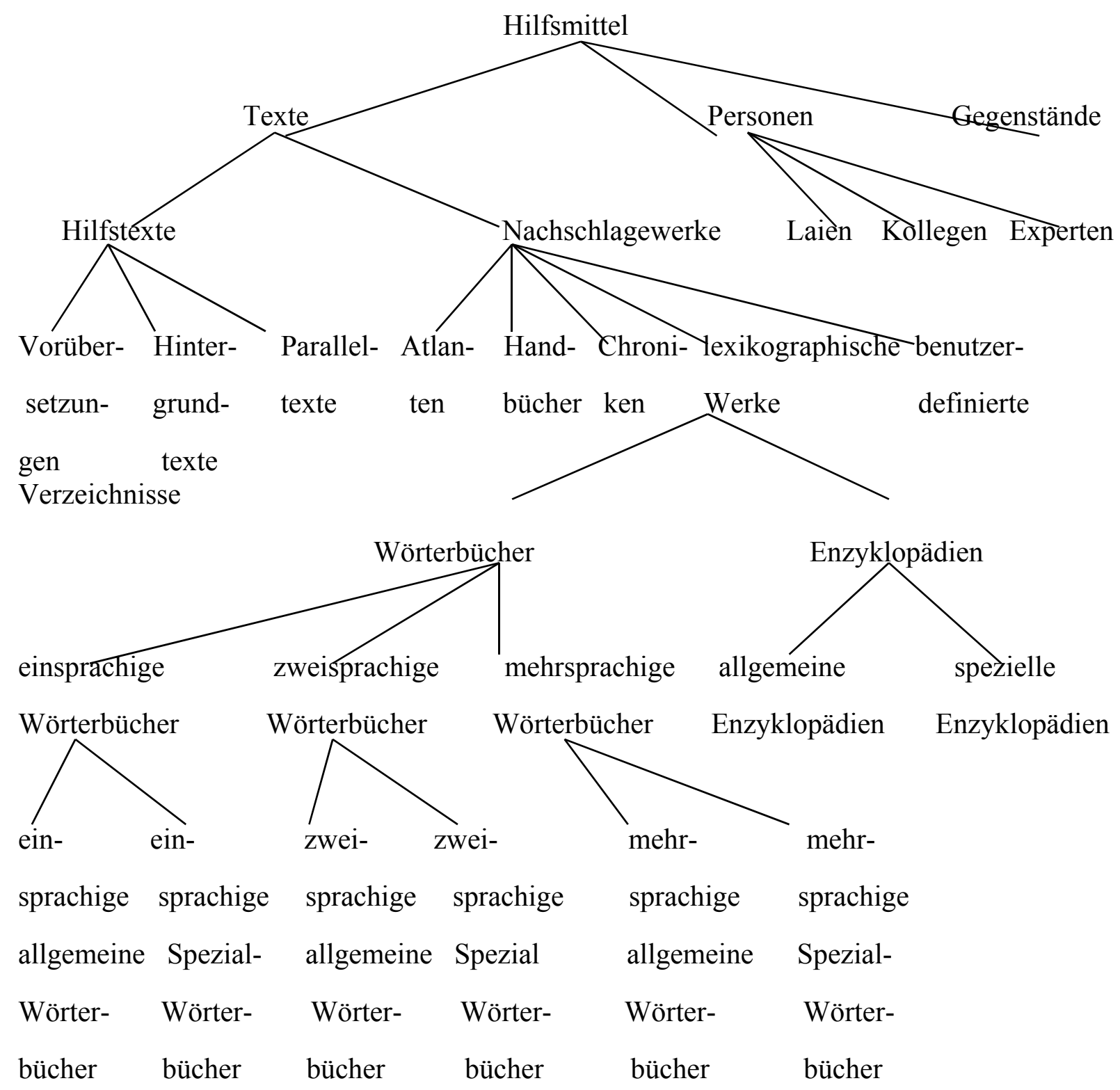

Abb. 1. Wiesmann, 2004, 158

Dass diese Hilfsmittel beim Übersetzen benutzt werden, erfordert von dem Übersetzer angesichts der Mühe und Zeit, dass er sich heute mehr als früher bemühen muss. Denn die Klassifizierung der verwendeten Nachschlagewerken, das Recherchieren über den Ausgangstext, die Verwendung der mehrsprachigen Wörterbüchern oder die Verhandlung mit den Fachleuten stellen den Übersetzer vor große Herausforderungen. Insbesondere der Zeitfaktor ist hier zu nennen.

\subsection{Die mündliche Übersetzung}


Wenn wir die Auffassungen von Şahin zitieren, kann man sagen, dass (Galiba, 1998) sich die Geschichte der simultanen mündlichen Übersetzung bis zu den Nürnberger Prozessen zurückführen lassen, die nach Ende des 2. Weltkrieges stattgefunden haben. Die in diesem Zeitraum verwendete Technologie bestand nur aus einem Kabelsystem. Dank dieses Systems, das sich aus Mikrofon, Kabel, Lautsprecher und Kopfhörer zusammensetzte, konnte man die spontane Übersetzung über vier getrennte Kanäle ausführen (für mehr technische Information vgl. Galiba, 1998, s. 68-9). Von dem Ende der 1940er Jahren bis heute ist es möglich zu sagen, dass sich die beim mündlichen Übersetzen benutzten Technologien nicht so weit änderten wie beim schriftlichen Übersetzen. Trotzdem muss man hinzufügen, dass zusammen mit den Entwicklungen im Bereich Computer und Kommunikation auch schon einige Veränderungen und Modifizierungen beim mündlichen Übersetzen stattgefunden haben. (Şahin, 2013: 4-5).

Diese Hilfsmittel, die für die mündlichen Übersetzungsanwendungen benutzt werden, tragen zu dem Übersetzungsprozess bei. Jedoch wie Şahin bezeichnet hat, ist der Wandel bei den mündlichen Übersetzungshilfsmittel nicht vielseitiger als bei den Schriftlichen. Die heutige Verwendung der traditionellen Hilfsmitteln kann man dafür als Beispiel geben. Abgesehen davon haben die Entwicklungen bei den Kommunikationsmitteln die Übersetzungstechnologien beeinflusst und auch die Hilfsmittel beim mündlichen Übersetzen beeinflußt. Abschließend kann man hier behaupten, dass die herkömmlichen Übersetzungshilfsmittel je nach aktuellen Möglichkeiten reformiert und novelliert wurden.

\subsection{Die Übersetzungsbildung}

Die Entwicklungen der mündlichen und schriftlichen Übersetzungsbildung fanden nicht parallel statt. Eine wichtigen Ursachen war die Regierungspolitik. Die zu der Übersetzungsbildung angebotenen Möglichkeiten bestanden nur darin, dass die als schriftliche Texte erhaltenen Texte wieder als schriftliche Texte in eine zweite Sprache übertragen wurden. Die Hilfsmittel in diesem Zeitraum gingen nicht über Stift, Papier und Wörterbuch hinaus.

Şahin äußert, dass der Gebrauch neuer Technologien trotz der rasanten Entwicklungen im Technologiefeld sowohl bei der schriftlichen als auch mündlichen Übersetzungsbildung viele Jahre begrenzt waren. Die am meisten spürbare Veränderung im Technologiegebrauch bei der Übersetzungsbildung wird in der mündliche Übersetzung deutlich. Während bis zum Ende der 1990er Jahren benutzte und höchste Technologie in den meisten Übersetzerschulen die Tonaufnahmekassette und Overhead-Projektor darstellten, benutzt man heute weithin fortschrittlichere Technologien. Wenn man die schriftliche Übersetzungsbildung betrachtet, beschränkt sich hier der Technologiegebrauch höchstens auf die Powerpointpräsentation (Şahin, 2013: 5).

Trotzdem sind die Hilfsmittel beim mündlichen Übersetzen gemä $\beta$ der finanziellen und physikalischen Bedingungen unterschiedlich. Die Beschaffungskosten einiger mündlicher Übersetzungshilfsmitel führt dazu, dass die Übersetzungsabteilungen der Bildungseinrichtungen meistens nur die schriftliche Übersetzungstätigkeit anbieten. Deswegen werden die Fähigkeiten der Studenten im Bereich der mündlichen Schilderung und auch Übertragung in die Fremdsprache nur wenig geschult und überwiegend auf das schriftliche Übersetzen beschränkt. Die Verbesserung der Staatspolitiken in bezug auf das Übersetzen und die Unterstützung des Staates hinsichtlich der technologischen Hilfsmittel konnten diesbezüglich eine Abhilfe schaffen.

\section{Welche Faktoren haben Einfluß auf dem Wandel der herkömmlichen Übersetzungsauffassung?}


Indem zunächst die Faktoren, die beim Wandel der herkömmlichen Übersetzungsanschauung eine Rolle spielen, aus den Perspektiven von den verschieden Autoren aufgezeigt worden sind, sollen in diesem Abschnitt Folgerungen daraus gezogen werden. Anhand der erworbenen Daten werden die den Wandel beeinflussenden Faktoren im Verlauf des Aufsatzes eingeordnet werden.

Heutzutage führt die sich schnell entwicklende Informatik zu weiten Neuerungen auch in der Übersetzungswelt. Viele Technologieanwendungen, Softwares, die den als Kommunikationsexperten gesehenen Übersetzer ersetzen, die interkulturelle Kommunikation im einfachsten Niveau leisten, kommen heraus. Als Abschluss der Entwicklungen, die in den 1940er Jahren begonnen haben, wurden nach 1980, wenn auch auf einfachem Niveau, Übersetzungsprogramme entwickelt. Von diesen computergestützten Übersetzungsprogrammen wird bekanntlich heutzutage vor allem bei der Übersetzung technischer Texte im Übermaß Gebrauch gemacht. Durch das Internet beschleunigt sich der Informationsfluss unglaublich und diese Information, die sich über die Welt ausbreitet, muss von den Kommunikationsexperten, also den Übersetzern, in die betreffenden Sprachen übersetzt werden. Zusammen mit der sich entwickelnden Technologie erhöhen sich die Anforderungen alltäglich an das Übersetzen, was eine gesteigerte Arbeitsbelastung der Übersetzer mit sich bringt. Dementsprechend ermöglicht die sich entwickelnde Technologie anstatt der nur aus dem Papier und Stift bestehenden Hilfsmittel viele technologische Anwendungen (Meral, Us, 2011: 43).

Neben den neuen Technologien, die als Folge der technologischen Entwicklungen zur Übersetzungshandlung einen Beitrag leisten, haben die obigen Aussagen in Worte gefasst, dass diese technologischen Entwicklungen durch das Übersetzen in andere Sprachen übertragen werden, und es wird im Folgenden die Funktion der Übersetzung als Übermittler von Information besprochen. Also stimmt, dass die Entwicklungen durch das Übersetzen bekannt werden. Wenn der Übersetzer denn diese neuen Hilfsmittel während der Übersetzungshandlung benutzt, sichert er damit auch den technologischen Fortschritt des Übersetzungsprozesses. Außer diesem Beitrag des Übersetzens können verschiedene Standpunkte bezüglich der Definition des Berufsbildes des Übersetzers von Interesse sein.

Nach Austermühl und Kornelius (1997) sind mit der Entwicklung multimedialer Instrumentarien und den vielfältigen Zugängen zu elektronischen Kommunikationsforen dem Übersetzer und Dolmetscher heute völlig neue Hilfsmittel an die Hand gegeben. In einem Zeitraum von weniger als zwei Jahren hat sich die Hilfsmittelsituation der Sprach- und Kulturmittler grundlegend verändert. So erfahren vor allem die Lehrenden und Studierenden in den Studiengängen der Übersetzungs- und Dolmetschwissenschaft die nachhaltigen Auswirkungen des medientechnischen Paradigmenwechsels in allen Bereichen ihres wissenschaftlichen Handelns (Austermühl, Einhauser, Kornelius, 1998: 335).

Durch diesen technologischen Wandel, der vom Übersetzer erlebt wird, vermehrt sich seine Verantwortung. Er ist nun nicht nur eine den schriftlichen oder mündlichen Text in die Zielsprache übertragende Person, sondern er muss auch die Funktion dieser Übersetzungshilfsmittel kennen sowie technologische Kenntnisse haben, wie er welches Hilfsmittel bei welchem Text nutzen kann. Eigentlich kürzen diese Hilfsmittel den Übersetzungsprozess ab und ermöglichen es, mehr Übersetzungsprodukte auf den Übersetzungsmarkt zu bringen. Darüber hinaus bedeuten sie eine enorme Zeitersparniss, da der Übersetzer nicht mehr stundenlang Zeit mit Nachschlagen in Wörterbüchern verbringt. Wenn der Übersetzer bewusst und verantwortungsvoll mit diesen neuartigen 
Übersetzungstechnologien umgeht, werden sich diese Herausforderungen auf seinen Beruf positiv spiegeln.

Mit der Nutzung moderner elektronischer Kommunikationsmöglichkeiten, die die Internettechnologie bietet, allen voran E-Mail, schwindet die Bedeutung des Standorts des einzelnen Übersetzers. Er kann ortsunabhängig Aufträge annehmen und liefern, solange er Zugang zum weltweiten Datennetz hat. Ein Großteil der Übersetzer arbeitet heute als Telearbeiter. Einerseits, da dies dank moderner Technik problemlos möglich ist, andererseits wird dies durch den Trend zum Outsourcing in der Übersetzungs- und Lokalisierungsindustrie weitgehend vorgegeben (Willer, 2007: 29).

Das Gesagte über das Berufsbild des Übersetzers ist auch Anlass zur Sorge. Dass der Übersetzer hier als Telearbeiter angesehen wird, kann zur Mechanisierung des Übersetzungsprozesses führen. Werden also diese Veränderungen in den Übersetzungstechnologien den Übersetzerberuf beseitigen oder den Beruf wieder umgestalten? Die Antwort kann man nur einschätzen. Die Tage sind vielleicht sehr nahe, an denen der Übersetzer nur eine Rolle als Lektor im Übersetzungprozess übernehmen wird.

Die klassischen Recherchemittel sind Paralelltexte (s. Art. 50) und Nachschlagewerke, wobei bei letzteren primär an Wörterbücher (s. Art. 49) gedacht wird, aber auch die selbst erstellten Terminologiekarteien gehören hierzu. Seit den 70er Jahren findet die Terminologieverwaltung zunehmend mit Hilfe von Computern statt, zunächst (und in manchen Untermehmen auch heute noch) ausschliesslich grossrechnerbasiert. Mit der Einführung von PCs begann die Verlagerung der kartei-und grossrechnerbasierten Terinologiearbeit auf PC-basierte Terminologieverwaltungssysteme; schon im Jahre 1989 setzten 30\% der mit PC arbeitenden Übersetzer ihren PC auch zur Terminologieverwaltung ein (Schmitt, 2006: 188-189).

Dies Zitat von Schmitt zeigt uns einen anderen Aspekt der in Übersetzungstechnologien erlebten Veränderungen. Es wird hier berichtet, dass die Terminologieinformation, die für die Übersetzungstätigkeiten sehr relevant ist, mit der Zeit mehr und mehr mithilfe von Computerprogrammen bewerkstelligt wird. Der Übersetzer hat jetzt die Möglichkeit, mit einem Klick Terminologiebanken zu erreichen und sich von den Terminologiebüchern und -karteien zu befreien. Diese Datenbanken ermöglichen dem Übersetzer die Wahrung der Terminologieeinheitlichkeit vor allem für Fachübersetzungen und gleichzeitig, dass der Übersetzer das Terminologieäquivalent in der Fremdsprache findet. Weil die Fachbereiche über unterschiedliche Terminologien verfügen, musste der Übersetzer früher oft Überstunden machen, die ihm heute durch die Terminologiedateien abgenommen werden.

Mit den Speicherschreibmaschinen in den 80er Jahren wurden die Möglichkeiten dieses Arbeitsmittels immer vielfältiger, doch spielen sie im Vergleich zu PCs bei Übersetzern heute nur noch eine marginale Rolle (Schmitt, 2006: 188).

Während Schmitt den Computer erörtert, den er als ein dem Übersetzer helfendes Mittel behandelt, vergleicht er ihn mit herkömmlichen Hilfsmitteln. Wenn wir dagegen die heutige Position des Computers und seine fast Unentbehrlichkeit thematisieren, wird die Unzulänglichkeit herkömmlicher Arbeitsmittel deutlich. Deshalb kann gesagt werden, dass der hinsichtlich der Technologie erlebte Paradigmenwechsel einer der praktischen Veränderungen ist.

In den 1980er Jahren war es das Ziel der US-amerikanischen Softwarehersteller, ihre Produkte an regionale Märkte und lokale rechtliche Vorschriften anzupassen. Hauptgrund dafür war die 
Steigerung des Verkaufs. In vielen Ländern bevorzugte man nämlich die Verwendung von Software in der Landessprache und manchmal ist auch aus rechtlichen Gründen eine Anpassung an die lokale Sprache von importierten Hard- und Softwareprodukten zusammen mit ihren Anleitungen erforderlich. Zu Anfang haben sich interne Abteilungen oder freiberufliche Übersetzer mit der Übersetzung von Softwareprodukten beschäftigt. Dann stellten Softwarehersteller mit der Zeit fest, dass nur ein sorgfältig geplantes Lokalisierungsprojekt den Erfolg eines Produkts garantieren konnte, allerdings fehlte das nötige Fachwissen dafür. Demnach haben sich Softwarehersteller für ein outsourcing model (vgl. Esslink 2000: 5) entschieden und multilinguale Anbieter übernahmen das gesamte Lokalisierungsprojekt. Diese Unternehmen haben sich mit der Zeit auf andere Bereiche spezialisiert, wie Desktop Publishing oder Software Engineering, da Softwarehersteller diese Aufgaben wegen mangelnder Sprachkenntnisse nicht mehr erledigen konnten. Bald boten sie ihre Dienstleistungen als Lokalisierungsdienstleistungen an (Baretta, 2013: 75).

Es ist ja klar, dass solche Veränderungen bei den Übersetzungshilfsmitteln den Übersetzungsmarkt befruchten. Die Bemühungen zur Verbesserung der Qualität von den Übersetzungsunternehmen und vor allem Softwareherstellern fördern kulturelle Forschungen. Hierzu bringen die Softwarehersteller den Begriff "Lokalisierung" auf die Tagesordnung, um das Interesse für das Übersetzungsprodukt zu erhöhen. Wie es in obigem Paragraph erklärt wird, erhöht die Lokalisierungsindustrie den Erfolg des Produkts und die vom Adressat im Übersetzungsmarkt angeforderte Menge.

Zusammenfassend können Kulturspezifik, neue Aufgaben für den Übersetzer, Übersetzungstechnologien, der Bedarf an einem professionellen Kompetenzprofil und die Spezialisierung zu den relativ neuen Trends der Fachübersetzung im Globalisierungszeitalter gezählt werden (Barretta, 2013: 117).

\section{Die Verwandlungen und Entwicklungen in Übersetzungshilfsmittel}

In heutiger Zeit, in der der Informationsaustausch durch die Computer in übernommen wird, kommen die Veränderungen der Übersetzung meistens dadurch zustande, dass die Übersetzungswerkzeuge funktionaler werden. Dass die Übersetzungshandlung mit einer die Zielgruppe und ihre kulturellen Eigenschaften berücksichtigenden Einstellung verfolgt wird, indem sie von der traditionellen Einsicht Abstand nimmt, ist entscheidend für die Rahmenbedingung der Übersetzungstechnologien. Die mit dieser Ausrichtung vorbereiteten Übersetzungsprogramme haben Hardwares, die auf die Aufmerksamkeit der Zielgruppe gerichtet sind. Es bietet dabei dem Übersetzer die Bequemlichkeit, dass die kulturellen Ausdrücke durch diese Werkzeuge übertragen werden können. Mit Hilfe der Übersetzungstechnologie kann der Übersetzer vielseitige Werkzeuge ganz einfach nutzen. Gleichwohl macht diese Vielfalt der Übersetzungshilfsmittel uns keine Sorgen, ob sich das Übersetzerprofil ändert oder nicht.

Nach Best und Kalina hat die rasante Entwicklung im Bereich der Informationstechnologie auch vor den Tätigkeiten des Übersetzens und Dolmetschens nicht Halt gemacht. Der Einsatz von computergestützten Hilfsmitteln ist aus dem Alltag des Übersetzens und Dolmetschens nicht mehr wegzudenken. Dieser Entwicklung hat sich die Ausbildung gestellt und auch die wissenschaftlichen Grundlagen des Übersetzens und Dolmetschens haben diesen Veränderungen bereits Rechnung getragen (Best, Kalina, 2002: 85).

Es ist von großer Bedeutung, dass der Übersetzer die aktuellen Hilfsmittel kennt und sie im Übersetzungsprozess aktiv verwendet, um im Informationszeitalter effizient zu sein. Die Übersetzungswerkzeuge, deren Erfindung den menschlichen Verstand beschleunigen, ersetzen 
den Übersetzer, der stundenlang mit Papier und Stift an einem Tisch arbeitete. Dementsprechend kann man auch davon ausgehen, dass das Verschwinden der klassichen Hilfsmittel davon herrührt. Nun ist es eine Realität, dass die Computer, die schneller als Menschenpotential sind und umfangreichere Systeme haben, zu übersetzen beginnen. Ihre Übersetzungen treten als maschinelle Übersetzung, Computer-aided Übersetzung, Übersetzung mit dem Computer, Computersoftwares für den Übersetzer, Dateienbanken, Terminologiebanken usw. in Erscheinung. Diese vom Menschen geschaffenen Programme und Softwares deuten uns zweifellos den Vorteil der Technologie an. Durch solche Softwares kann man den Übersetzungsprozess zufolge der eingetragenen Daten anfangen. Die OnlineSoftwares und Programme haben Qualifikationen, die es uns ermöglichen, dass wir die Wörter in anderen Sprachen mit der Ausgangsprache vergleichen können.

Also verpflichtet der neue und rasante Wandel im Bereich der Übersetzungshilfsmittel den Übersetzer mit ihnen mitzuhalten. Er soll jetzt von den Möglichkeiten der globalen Hilfsmittel zu Lasten der traditionellen Verfahren Gebrauch machen. Dementsprechend berichtet Kautz in dem folgenden Paragraph über das sich ändernde Profil des Übersetzens und Dolmetschens.

"Übersetzen und Dolmetschen haben in jüngerer Vergangenheit eine neue Bedeutung in der internationalen Kommunikation erlangt. Die explosionsartige Entwicklung des Übersetzungsund Dolmetschwesens ist vor allem dem Umstand zuzuschreiben, dass neben die traditionellen Bereiche des Übersetzens-z.B. Bibelübersetzung und literarisches Übersetzen- das fachsprachliche Übersetzen und das Dolmetschen von Fachveranstaltungen getreten ist: Sie haben heute die Dimension eines internationalen Massenkommunikationsmittels erreicht und sind eine wesentliche Vorbedingung für das reibungslose Funktionieren des Informationsaustausches auf wissenschaftlichem, technischem, wirtschaftlichem, politischem, soziokulturellem und militärischem Gebiet geworden. " (Bundesverband der Dolmetscher und Übersetzer [BDÜ] 1988, S. 1. ) (Kautz, 2002: 17).

Bevor wir zum anderen Teil, in dem die Übersetzungshilfsmittel anschaulicher behandelt werden, weitergehen, ist es erforderlich, Willers Aussagen zuzustimmen, der die Informationen über die Veränderungen und Entwicklungen wie folgt kurz zusammenfasst: Demnach wird davon ausgegangen, dass der Übersetzer mit diesen neuen Mittel das Übersetzungsprodukt sowohl schneller als auch qualifizierter herstellen kann.

Mehr und mehr konnten Texte beliebig reproduziert und archiviert werden. Die Arbeitsweise des Übersetzers und die Art und Weise, wie er seine Aufträge erhält, haben sich durch diese Entwicklung und die oben beschriebene fortschreitende Vernetzung der Computer in den letzten Jahren rasant und nachhaltig verändert, auch dank der vielen Systeme, die dem Übersetzer als elektronische Hilfsmittel zur Verfügung stehen. Vor allem Datenbanksysteme und sogenannte Übersetzerarbeitsumgebungen sind neben immer besseren Programmen zur Textverarbeitung aus dem heutigen Alltag eines Übersetzers nicht mehr wegzudenken. Diese Entwicklung trug neben weiteren produktivitätssteigernden Faktoren wie beispielweise dynamischen, fachlich kompetenten Teams dazu bei, dass die für eine konsistente, qualitativ hochwertige Übersetzung zu kalkulierende Zeitspanne gesunken ist (Willer, 2007: 50).

\section{Die heutigen Übersetzungshilfsmittel und ihr Eindruck auf den Übersetzungsprozess}

Die heute benutzten Übersetzungshilfsmittel entwickeln sich parallel zu den Technologien. Deswegen dienen die aufgelisteten Hilfsmittel ihren Funktionen nach für verschiedene Zwecke. Diese Werkzeuge, denen wir vor allem beim mündlichen und schriftlichen Übersetzen begegnen, werden entsprechend der Bedürfnisse des Adressaten erstellt und sie weisen uns von 
der Vergangenheit bis zur Gegenwart den letzten Punkt der Übersetzung. Als Beispiel für die Hilfswerkzeuge soll zunächst die Einordnung von Kornelius in Augenschein genommen werden.

In der Hilfsmittelkunde der Übersetzungswissenschaft hat sich dabei in den letzten Jahren eine Art von Kanon an elektronischen Hilfsmitteln herausgebildet und etabliert. Das Spektrum der sog. tools umfasst elektronische Wörterbücher, Enzyklopädien, fachbezogene elektronische Textkorpora, Sprach- und Fachdatenbanken, Textanalyseprogramme, Terminologieverwaltungs- und Translation Memory-Systeme und alle Formen der maschinengestützten digitalen Wissensrecherche in virtuellen Datennetzen und Datenbanken des Internets sowie der Nutzung elektronischer Kommunikationsforen und -formen wie $E$ Mail, Internet Relay Chat, Newsgroups (Kornelius, 2004: 437).

Wie gesehen behandelt Kornelius die Übersetzungshilfsmittel hinsichtlich elektronischer Quellen und stellt die heraus, die als kommunikationsorientiert verwendet werden. Während diese im Zentrum der Kommunikation benutzten Hilfsmittel den Übersetzungsprozess erleichtern, dienen sie gleichzeitig als Nebeneffekt der Aufgabe der Sprachenvermittlung durch Übersetzer. Wenn wir die tausenden auf der Welt gesprochenen Sprachen überlegen, wird die Übersetzung eines der Mittel, das vorrangig dafür sorgt, dass wir über diese Sprachen Kenntnis haben. Tatsächlich äußert Barretta darüber folgendes:

Heutzutage werden etwa 6000 Sprachen in der Welt gesprochen (vgl. Motz 2007). Unter den verschiedenen Möglichkeiten zur Überwindung der Sprachenvielfalt wird hier der Fokus auf die Institutionalisierung einer Weltverkehrssprache und später auf die Sprachmittlung durch Übersetzer gesetzt (Barretta, 2013: 55).

Nachdem das Berufsbild des Übersetzers und einige seiner Übersetzungshilfsmittel kürzlich behandelt worden sind, kann man sie jetzt nach ihren Funktionen folgendermaßen ordnen:

1. Hilfsmittel zur physischen Ausübung

- Tisch, Stuhl u.s.w reduzieren die physische Belastung

- Beleuchtung, Bücherregale, Aktenschränke, Dokumentenhalter... $=$ Grundausstattung des Arbeitsplatzes

2. Hilfsmittel zur Verwaltung

- Aktenordner, Computersoftware, Buchhaltungsprogramme helfen bei der Bewältigung der Verwaltungsaufgaben (z.B. der Steuererklärung)

3. Hilfsmittel zur Kommunikation

- Telefon, Faxgerät, Modem, Online-Dienste ermöglichen die Kommunikation mit anderen am Übersetzungsprozess Beteiligten

4. Hilfsmittel zur Textproduktion

$=$ Werkzeuge für die Textfixierung

- Papier, Schreibmaschine, Diktiergerät, Computerhardware (z.B. Speichermedien), Computerperipherie (z.B. Tastatur\&Bildschirm), Computersoftware (z.B.

Textverarbeitungsprogramm)

5. Hilfsmittel zur Recherche

- Ergänzung der Wissensbestände

- Zugriff auf externe Wissensbestände = Printmedien und elektronische Datensammlungen

- Wörterbücher, Paralleltexte, Weitere Technische Hilfsmittel, Relevanz der Hilfsmittel in der Berufspraxis $<$ Softwarelokalisierung $>$ (nur für Hilfsmittel vgl. Nord, Britta, 2002).

Der Einfluss der Übersetzungshilfsmittel auf den heutigen Übersetzerberuf ist unwiderlegbar aber wie wirken sie sich etwa auf den Übersetzungsprozess aus? 
Translationswissenschaftlich betrachtet stellen die Entwicklung der neuen Informations- und Kommunikationstechnologie sowie die Einführung neuer technischer Hilfsmittel Aspekte von großem Interesse dar. Sie haben nicht nur den Translationsprozess tiefen Veränderungen ausgesetzt, sondern auch die darin involvierten Sprachen, die Translatorrolle als Experte der interkulturellen Kommunikation und seine Aufgaben. Schlagwörter wie Lokalisierung, Outsourcing und Technical Writing sind überall in der einschlägigen Literatur zu lesen und insbesondere im Zusammenhang mit der Fachübersetzung, vor allem im wirtschaftlichökonomischen Bereich (vgl. Austermühl 2001a: 62). Eine wichtige Rolle spielen auch die neuen Hilfsmittel, wie z.B. die Terminologieverwaltungssysteme oder die translation memories, die heute die Arbeit von Translatoren erleichtern (vgl. Seel 2008: 6ff). (Barretta, 2013: 63).

Dass die Entwicklungen in Kommunikationsmedien Veränderungen im Übersetzungsprozess herbeiführen und damit das Übersetzen in eine neue Dimension geht, wirkt sich positiv auf den Übersetzungsmarkt aus. Wenn man insbesonders an die technische Übersetzung denkt, die auf dem Übersetzungsmarkt eine feste Stelle hat, sieht man, dass sich die technische Übersetzung durch diese Hilfsmittel qualitativ besser realisieren lässt, und dass dies eine Terminologievereinheitlichung zur Folge hat. Diese mit technischen Daten ausgestatteten Hilfsmittel (z.B. Terminologiebanken) erleichtern die Aufgabe des Übersetzers, und auch Paralleltexte werden als Muster für technische Übersetzung benutzt. Auf diese Weise hat der Übersetzer die helfenden Möglichkeiten, dass er sowohl in der Recherchephase als auch in der Textproduktionsphase entsprechend der zielsprachigen Normen den Übersetzungstext verfassen kann.

Die Hilfsmittel, die nicht nur auf die technische Übersetzung, sondern auch auf jede Phase der mündlichen und schriftlichen Übersetzung Einfluss haben, spielen eine wichtige Rolle im Gleichgewicht von Angebot und Nachfrage. Je mehr zielgruppenorientierte Übersetzungen es gibt, desto größer wird die Nachfragen nach solchen Übersetzungen (und immer mehr fremde Welten erschließen sich dem Nachfrager.) und alle fremden Dinge beginnen bekannt zu sein.

Die anderen Vorteile und Einflüsse der Übersetzungshilfsmittel auf den Übersetzungsprozess zeigt Baretta wie folgt (vgl. Barretta, 2013):

- $\quad$ Größere Sichtbarkeit und stärkere Wahrnehmung des Übersetzens

- Marktwachstum der Sprachdienstleistungen

- Höheres Tempo

- $\quad$ Neue zu übersetzende Inhalte

- $\quad$ Neue Arbeitsmittel

Durch diese Einflüsse, die dem Übersetzungsprozess einen neuen Umfang bringen, kann der Übersetzer gerade dabei sowohl auf der nationalen als auch internationalen Ebene die Übersetzungshandlung vorantreiben. Damit hat er die Fertigkeiten, die Texte mit neuen Arbeitsmitteln in höherem Tempo zu übersetzen.

\section{Schlusswort}

Wenn wir die Aussagen über den Wandel des Übersetzens nochmals reflektieren, ist erkennbar, dass die technologischen und wissenschaftlichen Entwicklungen große Auswirkungen auf das Übersetzen haben. Darum wäre die Tätigkeit des Übersetzers heute kaum ohne 
Übersetzungstechnologien denkbar. Sie warten mit mehreren Vorteile auf, wie eine Erleichterung der Arbeit und eine ständige Aktualisierung der eigenen Datenbanken des Übersetzers.

Die Übersetzungshilfsmittel ermöglichen, dass sich die lexikographische Praxis entwickelt und die Nachfrage nach sprachlichen Dienstleistungen steigt. Weiterhin eröffnen sich durch die neuen Werkzeuge mehrdirektionelle Recherchemöglichkeiten, eine höhere Schnelligkeit bei der Recherche und Textproduktion, was insgesamt eine größere Effizienz bedeutet. Zudem lassen sich Texte heutzutage durch den Einsatz von PCs problemsloser bearbeiten, und Einarbeitungen in den Text sowie der Einsatz von Textbausteinen sind dadurch leichter möglich geworden.

Gleichzeitig hat sich jedoch auch das Berufsbild des Übersetzers durch die Verfügbarkeit neuer Übersetzungswerkzeuge verändert. Ausschließlich sprachliche Fähigkeiten und Fertigkeiten reichen heute nicht mehr aus, um auf dem Übersetzungsmarkt erfolgreich zu sein. Heute muss der Übersetzer darüber hinaus auch technologische Kenntnisse haben, um die neuen Technologien effizient nutzen zu können. Er muss über sprachlexikographisches und computertechnisches Wissen verfügen, um Recherchen durchführen zu können, und Textverarbeitungsprogramme bedienen können. Diese neuen Anforderungen an den Beruf des Übersetzers stellen auch neue Herausforderungen an das Ausbildungsprofil an Dolmetscherschulen und an den Studiengang Übersetzungswissenschaften an Universitäten. Nach wie vor müssen natürlich sprachliche Kompetenz (in der eigenen und in der Fremdsprache) und theoretisches Grundwissen das solide berufliche Fundament bieten. Dafür ist es notwendig, während der Ausbildung fortlaufend mutter- und fremdsprachlich geschult zu werden, um in beiden Sprachen die Spracharchitektur zu beherrschen. Darüber hinaus müssen Studenten aber auch an die neuen technologischen Übersetzungswerkzeuge herangeführt werden, um auf dem hart umkämpften Übersetzungsmarkt bestehen zu können. Es sollte deshalb nie vergessen werden, dass Technologien immer nur so nützlich sind und nur so gute Ergebnisse erzielen, wie der Nutzer sie zu nutzen weiß. 


\section{References \\ Literaturverzeichnis}

- Austermühl, F., Einhauser, E., \& Kornelius, J. (1998). Die elektronischen Hilfsmittel des Übersetzers. URL: http://www. iued. uni-heidelberg.

de/institut/abteilung/englisch/areas/inmediasres/Areas15/41sprach. htm.(Zugriff: 16.4. 2005).

- Barretta, G. (2013). Globalisierung und Fachübersetzung (Doctoral dissertation, uniwien).

- Best, J. Kalina, S. (Hrsg). (2002). Übersetzen und Dolmetschen. A. Francke Verlag Tübingen und Basel.

- Kautz, U. (2002). Handbuch Didaktik des Übersetzens und Dolmetschens Goethe Institut und Iudicium Verlag..

- Kornelius, J. (2004). Texten und Übersetzen in vernetzten Wissenskonstruktionen. AREAS-Annual Report on English and American Studies, 27, 435-454.

- Meral, H. Us, M. (2011) “Çeviri Ĕgitiminde Bilgi Teknolojilerinin Önemi” TÜÇEB I. ULUSAL ÇEVİRİBİLIM ÖĞRENCİ ÇALIŞTAYI, Hacettepe Üniversitesi, Ankara, Çeviri Ë̆itimi, 42-51.

- Nord, B. (2002). Hilfsmittel beim Übersetzen. Eine empirische Studie zum Rechercheverhalten professioneller Übersetzer. Frankfurt aMua: Lang

- Schmitt, P.A. (2006). Technische Arbeitmittel. Handbuch Translation. Stauffenburg Verlag.

- Şahin, M. (2013). Çeviri ve Teknoloji. İzmir Ekonomi Üniversitesi Yayınları, İzmir.

- Wiesmann, E. (2004). Rechtübersetzung und Hilfsmittel zur Translation. Gunter Narr Verlag Tübingen.

- Willer, F. (2007). Übersetzen und Globalisierung. BDÜ Fachverlag. 